\title{
Functions preserving rank- $k$ matrices of order $n$ over fields
}

\author{
Lizhu Hao and Xian Zhang
}




\title{
FUNCTIONS PRESERVING RANK- $\boldsymbol{k}$ MATRICES OF ORDER $\boldsymbol{n}$ OVER FIELDS
}

\author{
LIZHU HAO AND XIAN ZHANG
}

[Received: June 16, 2004]

\begin{abstract}
Aвstract. Let $\mathbb{F}$ be an arbitrary field and $n$ is an integer with $n \geq 2$. For a fixed positive integer $k$ satisfying $k<n$, we determine the general form of all functions preserving rank- $k$ matrices of order $n$. This article generalizes the recent results of J. Kalinowski [1,2].

Mathematics Subject Classification: 15A03
\end{abstract}

Keywords: field, rank- $k$ matrix, function

\section{INTRODUCTION}

Suppose $\mathbb{F}$ is an arbitrary field and $\mathbb{R}$ is the field of the real numbers. Let $n$ be an integer with $n \geq 2$. For a function $f: \mathbb{F} \rightarrow \mathbb{F}$ and a matrix $A=\left[a_{i j}\right]$ over $\mathbb{F}$, denote the matrix $\left[f\left(a_{i j}\right)\right]$ by $A^{f}$. We say that a function $f: \mathbb{F} \rightarrow \mathbb{F}$ preserves ranks of matrices if $\operatorname{rank} A^{f}=\operatorname{rank} A$ for all matrices (of any order) over $\mathbb{F}$, and preserves rank- $k$ matrices of order $n$ if $\operatorname{rank} A^{f}=\operatorname{rank} A$ for every rank- $k$ matrix of order $n$.

Kalinowski [1] investigated that a monotonic and continuous function $f: \mathbb{R} \rightarrow \mathbb{R}$ with $f(0)=0$ preserves ranks of matrices if and only if it is linear, i. e., $f(x)=c x$ for every $x \in \mathbb{R}$ and some non-zero $c \in \mathbb{R}$. Furthermore, in [2], Kalinowski generalized the result in [1] by removing any restrictions on the function $f$.

Inspired by [1,2], in this article we prove the following two theorems which generalize the result in [2].

Theorem 1. Let $k$ be a fixed integer satisfying $2 \leq k<n$. Then $f: \mathbb{F} \rightarrow \mathbb{F}$ is a function preserving rank- $k$ matrices of order $n$ if and only if there exist a non-zero scalar $c$ and an injective field endomorphism $\delta$ of $\mathbb{F}$ such that $f=c \delta$.

The first author was supported in part by the NSF of Heilongjiang Education Committee, Grant No. 15011014

The second author was supported in part by the Chinese Natural Science Foundation, Grant No. 10271021, the Natural Science Foundation of Heilongjiang Province, Grant No. A01-07, and the Fund of Heilongjiang Education Committee for Overseas Scholars, Grant No. 1054HQ004. 
Theorem 2. $f: \mathbb{F} \rightarrow \mathbb{F}$ is a function preserving rank-1 matrices of order $n$ if and only if either $f$ is a non-zero constant function or $f=c \kappa$, where $c$ is a non-zero scalar and $\kappa: \mathbb{F} \rightarrow \mathbb{F}$ is a multiplicative function with $\kappa(0)=0$ and $\kappa(1)=1$.

As pointed out by Marková [3], these results obtained in [1,2] play a important role in the theory of $g$-calculus (see [4] for the concept of $g$-calculus and the relevant topics). Therefore, Theorems 1 and 2 will be helpful for studying extensively $g$ calculus.

We end this section by introducing the notation which will be used in the next section. Denote by $\oplus$ the usual direct sum of matrices. For a positive integer $k$, let $I_{k}$ be the $k \times k$ identity matrix over $\mathbb{F}$

\section{Proofs of Theorems 1 and 2}

The Proof of Theorem 1. The "if" part is obvious. The proof of the "only if" part is divided into the following four steps.

Step 1: $f(0)=0$ and $f(d) \neq 0$ for every non-zero scalar $d$. For any non-zero scalar $d$, it follows from rank $\left(d I_{k} \oplus 0\right)=k$ and the definition of $f$ that

$$
\operatorname{rank}\left[\begin{array}{cccccc}
f(d) & f(0) & \cdots & \cdots & \cdots & f(0) \\
f(0) & \ddots & \ddots & & & \vdots \\
\vdots & \ddots & f(d) & \ddots & & \vdots \\
\vdots & & \ddots & f(0) & \ddots & \vdots \\
\vdots & & & \ddots & \ddots & f(0) \\
f(0) & \cdots & \cdots & \cdots & f(0) & f(0)
\end{array}\right]=k,
$$

where the number of occurrences of $f(d)$ is equal to $k$. This, together with the inequality $2 \leq k<n$, completes the present step.

Step 2: $f(1) f(x y)=f(x) f(y)$ for all $x, y \in \mathbb{F}$. For any $x, y \in \mathbb{F}$, since

$$
\operatorname{rank}\left(\left[\begin{array}{cc}
1 & x \\
y & x y
\end{array}\right] \oplus I_{k-1} \oplus 0\right)=k
$$

it follows from Step 1 (i. e., $f(0)=0$ ) and the definition of $f$ that

$$
\operatorname{rank}\left(\left[\begin{array}{cc}
f(1) & f(x) \\
f(y) & f(x y)
\end{array}\right] \oplus f(1) I_{k-1} \oplus 0\right)=k,
$$

and hence

$$
\operatorname{det}\left(\left[\begin{array}{ll}
f(1) & f(x) \\
f(y) & f(x y)
\end{array}\right] \oplus f(1) I_{k-1}\right)=0
$$

By direct computation, one shows that $f(1)^{k-1}(f(1) f(x y)-f(x) f(y))=0$. This, together with Step 1 (i. e., $f(1) \neq 0$ ), gives $f(1) f(x y)=f(x) f(y)$. 
Step 3: $f(x+y)=f(x)+f(y)$ for all $x, y \in \mathbb{F}$. For any $x, y \in \mathbb{F}$, in view of the relation

$$
\operatorname{rank}\left(\left[\begin{array}{ccc}
0 & 1 & x \\
1 & 0 & y \\
1 & 1 & x+y
\end{array}\right] \oplus I_{k-2} \oplus 0\right)=k,
$$

it follows from Step 1 (i. e., $f(0)=0$ ) and the definition of $f$ that

$$
\operatorname{rank}\left(\left[\begin{array}{ccc}
0 & f(1) & f(x) \\
f(1) & 0 & f(y) \\
f(1) & f(1) & f(x+y)
\end{array}\right] \oplus f(1) I_{k-2} \oplus 0\right)=k .
$$

Furthermore,

$$
\operatorname{det}\left(\left[\begin{array}{ccc}
0 & f(1) & f(x) \\
f(1) & 0 & f(y) \\
f(1) & f(1) & f(x+y)
\end{array}\right] \oplus f(1) I_{k-2}\right)=0 .
$$

Thus, $f(1)^{k}(f(y)+f(x)-f(x+y))=0$. This, together with Step 1 (i. e., $f(1) \neq 0$ ), implies that $f(x+y)=f(x)+f(y)$.

Step 4: there exist a non-zero scalar $c$ and an injective field endomorphism $\delta$ of $\mathbb{F}$ such that $f=c \delta$. If we denote $c=f(1)$ and $\delta=c^{-1} f$, then $f=c \delta$ and $c$ is a non-zero scalar. Furthermore, it is easy to verify from Steps $1-3$ that $\delta$ is an injective field endomorphism of $\mathbb{F}$.

The proof is complete.

Proof of Theorem 2. The "if" part. If $f$ is a non-zero constant function, then, clearly, $f$ preserves rank-1 matrices of order $n$.

Now we prove the case $f=c \kappa$, where $c$ is a non-zero scalar and $\kappa: \mathbb{F} \rightarrow \mathbb{F}$ is a multiplicative function with $\kappa(0)=0$ and $\kappa(1)=1$. For an arbitrary rank-1 matrix $A$, it can be written as $A=\left[a_{i} b_{j}\right]$, where $a_{i}, b_{i} \in \mathbb{F}, i=1, \cdots, n$, and $a_{p} b_{q} \neq 0$ for some $p, q$. Hence $A^{f}=\left[c \kappa\left(a_{i} b_{j}\right)\right]=c\left[\kappa\left(a_{i} b_{j}\right)\right]$. Since $\kappa$ is multiplicative, it can be concluded that $A^{f}=c\left[\kappa\left(a_{i}\right) \kappa\left(b_{j}\right)\right]$, which implies rank $A^{f} \leq 1$. On the other hand, for any nonzero $d \in \mathbb{F}$, it follows from $d d^{-1}=1$ and the multiplicativity of $\kappa$ that $\kappa(d) \kappa\left(d^{-1}\right)=$ $f(1)$. Using $\kappa(1)=1$, we have $\kappa(d) \neq 0$. Therefore, $\kappa\left(a_{p}\right) \kappa\left(b_{q}\right)=\kappa\left(a_{p} b_{q}\right) \neq 0$ since $a_{p} b_{q} \neq 0$. In summary, rank $A^{f}=1$, i. e., $f$ is a function preserving rank-1 matrices of order $n$.

The "only if" part. For any non-zero scalar $d$, it follows from rank $(d \oplus 0)=1$ and the definition of $f$ that

$$
\operatorname{rank}\left[\begin{array}{cccc}
f(d) & f(0) & \cdots & f(0) \\
f(0) & f(0) & \ddots & \vdots \\
\vdots & \ddots & \ddots & f(0) \\
f(0) & \cdots & f(0) & f(0)
\end{array}\right]=1
$$


Case 1. Suppose that $f(0) \neq 0$. Then, by (2.1), we have $f(d)=f(0)$. Since $d$ is an arbitrary non-zero scalar, we can claim that $f$ is a non-zero constant function.

Case 2. Suppose that $f(0)=0$. Then, by (2.1), we have $f(d) \neq 0$ for any non-zero scalar $d$. For any $x, y \in \mathbb{F}$, since

$$
\operatorname{rank}\left(\left[\begin{array}{cc}
1 & x \\
y & x y
\end{array}\right] \oplus 0\right)=1
$$

it follows from $f(0)=0$ and the definition of $f$ that

$$
\operatorname{rank}\left(\left[\begin{array}{cc}
f(1) & f(x) \\
f(y) & f(x y)
\end{array}\right] \oplus 0\right)=1
$$

Thus,

$$
\operatorname{det}\left[\begin{array}{cc}
f(1) & f(x) \\
f(y) & f(x y)
\end{array}\right]=0
$$

i. e., $f(1) f(x y)=f(x) f(y)$. If we put $c=f(1)$ and $\kappa=c^{-1} f$, then $f=c \kappa$ and $c$ is a non-zero scalar. Furthermore, it is easily verified that $\kappa$ is multiplicative function from $\mathbb{F}$ to itself such that with $\kappa(0)=0$ and $\kappa(1)=1$. This completes the proof.

\section{REFERENCES}

[1] Kalinowski, J.: On rank equivalence and preserving rank operators, Novi Sad J. Math., 32 (2002), 133-139.

[2] Kalinowski, J.: On functions preserving rank of matrices, Math. Notes, Miskolc, 4 (2003), 35-37.

[3] Markoví, A.: Some remarks on the pseudo-linear algebra, Tatra Mountains Math. Publ., 6 (1995), 123-130.

[4] PAP, E.: g-calculus, Zb. Prir.-Mat. Fak., Univ. Novom Sadu, Ser. Mat., 23 (1993), 145-156.

\section{Authors' addresses}

\section{Lizhu Hao:}

Heilongjiang University, Department of Mathematics, 150080 Harbin, P. R. China

E-mail address: haolz@hlju.edu.cn

\section{Xian Zhang:}

Heilongjiang University, Department of Mathematics, 150080 Harbin, P. R. China

E-mail address: zhangx663@sohu.com; x.zhang@qub.ac.uk

Current address: Queen's University of Belfast, School of Mechanical and Manufacturing Engineering, Stranmillis Road, BT9 5AH Belfast, UK

E-mail address: zhangx663@sohu.com 